\title{
MONITORING OF ENVIRONMENTAL POLLUTION IN A WOODY FOREST IRRIGATED WITH CONTAMINATED WASTEWATER SUBJECTED TO A PRIMARY TREATED
}

\author{
Taha Abd El-Khalik El-Maghraby, Mohamed Adel Ahmed Bakry \\ and Nadia Abd El-HAzzem Mohamed \\ Soils, Water and Environ. Res. Institute, Agric. Res. Center, Giza, Egypt
}

\section{ABSTRACT:}

This study was aimed at monitoring the environmental pollution of a loamy sand soil cultivated with different tree woody species, i.e., Morus alba, Khaya senegalensis, Acacia saligna and Populus nigra and irrigated with treated contaminated wastewater through drip irrigation system. Some heavy metals, i.e, $\mathrm{Cd}, \mathrm{Co}, \mathrm{Ni}$ and $\mathrm{Pb}$ were determined in each of irrigation water, irrigated soil and grown woody trees, whether the expected phytoremediators were able to accumulate and translocate these heavy metals, from lower plant part tissues to the upper ones, taking into consideration their concentrations in irrigation water and irrigated soil. In order to achieve this target, two areas were selected in Egypt, i.e., a) Egyptian Chinese friendship woody forest of the previous four tree species at Sadat City, Minufiyah Governorate, Egypt which irrigated with a primary treated wastewater and b) Egyptian-Japanese woody forest at Wadi AlNatron, Behaira Governorate, which irrigated with natural underground water, as a control for Acacia saligna only.

The obtained results showed an always seasonal difference as regards different studied heavy metal concentrations in irrigation water during 2010 that was noticeably higher than 2009. However, $\mathrm{Ni}$ and $\mathrm{Pb}$ concentrations in irrigation water of Sadat City area exceeded the permissible values of Egypt contaminated water. Also, $\mathrm{Ni}$ concentration in the natural underground water of Wadi Al-Natron area exceeded the permissible limit values of fresh water. As for heavy metal concentrations in the soil, data revealed that $\mathrm{Cd}$ concentrations were found in a low concentration at soil supporting Populus nigra vs a high concentration in subsurface layer $(20-40 \mathrm{~cm})$ in the soil supporting Acacia saligna. The highest concentration of $\mathrm{Ni}$ was occurred at subsurface layer (20-40 $\mathrm{cm}$ ) of soil supporting Acacia saligna and Khaya senegalensis. On the other hand, soil $\mathrm{Pb}$ and $\mathrm{Co}$ concentrations were almost similar for the four tree species.

Concerning the metal concentrations in tree species, there were insignificant differences for $\mathrm{Cd}$ concentrations in different tree stems, however, the different distribution pattern of Co concentrations in stem could be categorized in an ascending order of Populus $>$ Morus $>$ Khaya $>$ Acacia . Co and $\mathrm{Ni}$ concentrations in leaves were also taken an ascending order of Morus $>$ Khaya $>$ Acacia $>$ Populus. $\mathrm{Pb}$ concentrations in leaves were taken ascending order of Morus > Acacia > Khaya > Populus. All metals were successfully transported from roots to stems for all the investigated tree species. In Morus alba metals showed translocation preferences of $\mathrm{Pb}>\mathrm{Ni}>\mathrm{Co}>\mathrm{Cd}$ vs $\mathrm{Cd}>\mathrm{Pb}$ $>\mathrm{Ni}>\mathrm{Co}$ in Khaya, $\mathrm{Cd}>\mathrm{Ni}>\mathrm{Co}>\mathrm{Pb}$ in Acacia and $\mathrm{Pb}>\mathrm{Co}>\mathrm{Ni}>\mathrm{Cd}$ in Populus. In addition, Morus alba exhibited $\mathrm{Ni}$ and Co highest translocation factor values that were found only at the highest temperature degrees.

Moreover, $\mathrm{Ni}$ and Co concentrations in plant were found to be affected by soil $\mathrm{pH}$, organic matter and irrigation water salinity these may be assisting naturally to accelerate soil phytoremediation processes at warm climate under desert regions. So, it could be recommended that using Morus alba and Populus nigra for metal removal from soil is only technically feasible in short time period

Fayoum J. Agric. Res. \& Dev., Vol.25, No.1, January, 2011 
if more metal can be made available by adding chemicals to soil that increased their concentrations. It is essential in case of using Morus alba as a phytoremediator to discard any fruit or leaves, however, it is also recommended to study the effect of accumulated metals in Morus leaves on silk warm as a biological indicator. The metal amounts extracted by plants can be put into perspective by calculating a theoretically decrease in soil metal concentrations from the known plant maximum metal concentrations in order to calculate the concentration factor, i.e., $\mathrm{CF}=$ metal concentration in plant tissue/its concentration in the soil. This could be achieved according to the number of planted trees/fed. Also, it should be following up Ni concentration in the natural underground water at the Egyptian-Japanese forest to avoid Ni pollution.

Keywords: Woody forest, environmental pollution, phytoremediation of heavy metals.

\section{INTRODUCTION:}

Heavy metals contamination of soil and groundwater is widespread and hazardous to human and animal life. There are many sites need remediation all over the world. Phytoremediation is a viable, relatively low-cost approach to removing heavy metals from soil and groundwater (Salido et al., 2003). Heavy metals contamination occurs from a variety of sources. Sewage sludge treatments used as a fertilizer are major contributors to soil contamination (Do Nacimiento et al., 2006). Phytoremediation is the use of green plants to remove contaminants from soil and groundwater or to lower contaminant mobility by using phytoextraction, rhizofilteration, phytovolitization or phytotransformation (Madrid et al., 2003). Hyperaccumulators are plants that are able to take up large quantities of metals (Roosens et al., 2003). The classification of a hyperaccumulator is based on the ability to uptake and retain, within the stem and leaves (Turgut et al., 2005).

The cost of phytoremediation can be as low as $\$ 0.05$ per cubic meter $(\mathbf{L i}$, 2004). The total and available heavy metal concentrations of $\mathrm{Cd}, \mathrm{Ni}$, and $\mathrm{Pb}$ in the soil of the Egyptian Chinese forest at Sadat city, Menoufia Governorate, were found to be relatively high, their concentrations in the growing plant tissues, were over the allowable limits specially Ni (Salem, 2004). These metals were tested together for the best understanding of the plant response; Morus alba, Acacia salegna, Khaya senegalensis and Populus nigra are among the well growing woody species in the Egyptian Chinese forest. Most trace elements in water especially heavy metals do not exist in soluble forms for long time, they are present mainly as suspended in colloids or are fixed by organic and mineral substances. On average, 25,000 to 30,000 tons of cadmium is released into the environment each year (ATSDR, 1999). In Egypt the content of heavy metals in irrigation water was studied by many investigators, Abo El-Roos et al. (1996) reported that the use of wastewater in irrigation is the main source of heavy metals in agricultural soil in Egypt.

Concentration of heavy metals in waste water samples had wide variation from month to the other. Once the metals have been concentrated and extracted by the plants they can be easily removed from the site (Vervake $\boldsymbol{e t}$ al., 2006).

There are several plants mechanisms employed in reaction to exposure to contamination. These include phytoextraction, rhizofiltiration, phytovolatliz, Phyto-stabilization, phytotransformation and phytodegradation and aerial contaminant removal (Yang et al, 2005). Once the metals are available to the plant, they must be transported across the membrane of root cells, loaded into and translocate through the xylem and detoxified and stored within cells of the plant in

Fayoum J. Agric. Res. \& Dev., Vol.25, No.1, January, 2011 
a particular section (roots, leaves, stems). Nickel has shown 60-70\% storage in cell walls rather than vacuoles (Yang et al., 2005). There are three mechanisms that accompany heavy metal toxicity in plants; autoxidation and Fenton reaction production of reactive oxygen species blocking essential functional groups in the biomolecules and storage of essential metals ions from the biomolecules (Nouairi et al., 2006). Several factors affect metal uptake and distribution in plants, including physiochemical properties of contaminants, temperature, soil properties and plant characteristics (Gardea $\boldsymbol{e t}$ al., 2005). Plants are able to exude materials from roots to acidify soil and increase solubility and transports of contaminants (Suresh and Ravishankar, 2004). Do Nacimiento et al. (2006) showed a shoot selectivity of $\mathrm{Ni}>\mathrm{Cd}=\mathrm{Pb}$ without EDTA and $\mathrm{Ni}>\mathrm{Pb}>\mathrm{Cd}$ with EDTA.

This study was aimed at monitoring some heavy metals status in irrigation water, soil media and grown tree species in a natural woody forest irrigated with treated contaminated wastewater, with special reference to make a comparative study with un-contaminated woody trees as a control.

\section{MATERIALS AND METHODS}

This study was carried out on two selected areas, i.e., 1) Egyptian Chinese Friendship Woody Forest that were planted in 2001 at Sadat city, Menoufiah Governorate, $88 \mathrm{~km}$ northern- west of Cairo City and 2) Egyptian Japanese Woody Forest at Wadi Al-Natrun, Beheira Governorate, $150 \mathrm{~km}$ northern-west of Cairo City, Egypt. Such two areas represent a portion of the Northern African Sahara Region. The grown woody trees in the first area were directly irrigated with primary treated wastewater (an oxidation pond via drip irrigation), while the second one irrigated with the natural underground water. In general, soil texture of the selected two areas is loamy sand (sand $=80.33 \%$, silt $=17.23 \%$ and clay $=$ $2.44 \%)$. The two areas have an arid climate with a rare winter rainfall, some meteorological data for 2009 and 2010 are presented in Table (1).

Table (1): Meteorological data of Sadat City and Wadi Al-Natron Stations.

\begin{tabular}{|c|c|c|c|c|}
\hline \multirow{2}{*}{ Area } & Season & $\begin{array}{c}\text { Temperature }{ }^{0} \mathrm{C} \\
(\text { mean })\end{array}$ & $\begin{array}{c}\text { Relative } \\
\text { humidity } \%\end{array}$ & $\mathrm{ET}_{0}$ \\
\hline \multirow{4}{*}{ Sadat } & Spring 2009 & 9.26 & 70.67 & 3.19 \\
\cline { 2 - 5 } & Autumn 2009 & 23.33 & 65.89 & 4.19 \\
\cline { 2 - 5 } & Spring 2010 & 15.20 & 65.29 & 3.23 \\
\cline { 2 - 5 } & \multirow{2}{*}{ Autumn 2010 } & 26.30 & 70.10 & 4.60 \\
\cline { 3 - 5 } & 24.21 & 59.22 & 4.19 \\
\hline Wadi Al-Natron & &
\end{tabular}

Woody stands in the first area are included four tree species, i.e., Morus alba, Acacia salegna, Khaya senegalensis and Populus nigra. Soil characteristics at each species were studied at soil depths of 0-20, 20-40 and 40-60 cm. A comparison study was undertaken on the studied heavy metals absorb by Acacia trees under the same experimental conditions of the un-polluted area of Wadi Al-Natron. Thus, the essential materials in this investigation are the growing woody trees, their supporting soil, irrigation water samples from either oxidation pond at Sadat city or natural underground water at Wadi Al-Natron. The chosen investigated tree species of Morus alba, Acacia saligna, Khaya senegalensis and Populus nigra in the first area are belonging the families of Moraceae, Mimosaceae, Meliaceae and Salicaceae, respectively, while the second area comprises Acacia saligna.

Irrigation water samples were collected four times during the studied periods (spring and autumn of 2009 and 2010 seasons), transported immediately to the

Fayoum J. Agric. Res. \& Dev., Vol.25, No.1, January, 2011 
laboratory for analysis. Soil samples supporting investigated tree species were collected from both areas for analysis. Plant samples were taken from each area for analysis. Tables (2-5) showed some chemical properties and heavy metal concentrations in the studied irrigation waters and soils, which were determined according to the methods outlined by Jackson (1973). Soil particle size distribution and $\mathrm{CaCO}_{3}$ were determined according to Piper (1950). Soil organic matter was determined according to the method outlined by Black et al. (1965). Concentrations of $\mathrm{Cd}, \mathrm{Co}, \mathrm{Ni}$ and $\mathrm{Pb}$ in the used irrigation water as well as their available concentrations in soil were extracted according to Soltanpour (1985), and were measured using the Inductively Coupled Plasma Spectrometry (Plasma 400). Translocation factor of heavy metals was determined by dividing the total concentration in both stems and leaves by concentration in plant roots (Do Nacimiento et al., 2006). The obtained data were statistically analyzed by using the soft ware of SPSS statistical package of Social Science Version 13.

Table (2): Water chemical characteristics of the oxidation pond (Sadat) and natural underground water samples (Wadi Al-Natron) during studied seasons.

\begin{tabular}{|c|c|c|c|c|c|}
\hline Area & Season & $\mathrm{pH}$ & $\mathrm{EC}\left(\mathrm{dS} \mathrm{m}^{-1}\right)$ & SAR & Hardness ${ }^{*}$ \\
\hline \multirow{4}{*}{ Sadat } & Spring 2009 & 6.60 & 1.55 & 5.38 & 175 \\
\hline & Autumn 2009 & 7.40 & 1.76 & 3.90 & 295 \\
\hline & Spring 2010 & 6.90 & 1.67 & 3.78 & 295 \\
\hline & \multirow{2}{*}{ Autumn 2010} & 6.60 & 1.77 & 4.36 & 295 \\
\hline Wadi Al-Natron & & 7.20 & 1.20 & 11.9 & 80 \\
\hline
\end{tabular}

Table (3): Water concentrations of heavy metals (means \pm Sd) of a primary treated wastewater (Sadat) and natural underground water samples (Wadi AlNatron) during the studied seasons.

\begin{tabular}{|c|c|c|c|c|c|}
\hline \multirow{2}{*}{ Area } & \multirow{2}{*}{ Season } & \multicolumn{4}{|c|}{ Heavy metal contents $\left(\mathrm{mg} \mathrm{L}^{-1}\right)$} \\
\cline { 3 - 6 } & & $\mathrm{Cd}$ & $\mathrm{Co}$ & $\mathrm{Ni}$ & $\mathrm{Pb}$ \\
\hline \multirow{3}{*}{ Sadat } & Spring 2009 & $0.009 \pm 0.001$ & $0.016 \pm 0.001$ & $1.467 \pm 0.000$ & $0.011 \pm 0.000$ \\
\cline { 2 - 6 } & Autumn 2009 & $0.003 \pm 0.001$ & $0.0111 \pm 0.001$ & $1.660 \pm 0.000$ & $0.075 \pm 0.001$ \\
\cline { 2 - 6 } & Spring 2010 & $0.000 \pm 0.000$ & $0.053 \pm 0.001$ & $3.506 \pm 0.001$ & $4.123 \pm 0.000$ \\
\cline { 2 - 6 } & \multirow{2}{*}{ Autumn 2010 } & $0.144 \pm 0.001$ & $0.064 \pm 0.001$ & $1.781 \pm 0.000$ & $40.098 \pm 0.000$ \\
\cline { 3 - 6 } & & 0.003 & 0.076 & 0.989 & 0.007 \\
\hline Wadi Al-Natron & & & & &
\end{tabular}


MONITORING OF ENVIRONMENTAL POLLUTION IN.........

Table (4): Chemical properties of soil supporting tree species as affected by irrigation water in both areas during the studied seasons.

\begin{tabular}{|c|c|c|c|c|c|c|c|c|c|c|c|}
\hline \multirow[b]{2}{*}{ 总 } & \multirow[b]{2}{*}{$\begin{array}{l}\tilde{D} \\
0 \\
\tilde{D} \\
\tilde{n}\end{array}$} & \multirow{2}{*}{ 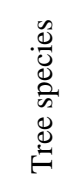 } & \multirow[b]{2}{*}{ 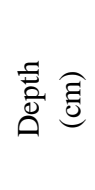 } & \multicolumn{4}{|c|}{2009} & \multicolumn{4}{|c|}{2010} \\
\hline & & & & $\mathrm{pH}$ & 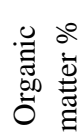 & 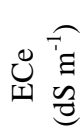 & $\underbrace{\infty}_{0} \therefore$ & $\mathrm{pH}$ & 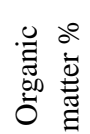 & 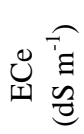 & $\bigcup_{\tilde{U}}^{\infty} \circ$ \\
\hline \multirow{18}{*}{ 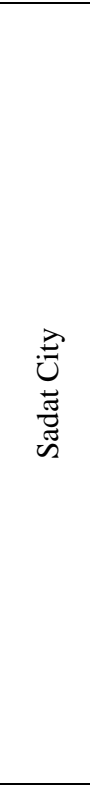 } & \multirow{3}{*}{ Spring } & \multirow{6}{*}{ 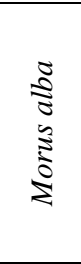 } & $0-20$ & 6.60 & 1.90 & \multirow{3}{*}{0.21} & 2.80 & 5.60 & 2.00 & \multirow{3}{*}{1.80} & 2.00 \\
\hline & & & $20-40$ & 6.90 & 1.70 & & 2.60 & 5.70 & 1.10 & & 2.00 \\
\hline & & & $40-60$ & 6.90 & 1.00 & & 2.40 & 5.70 & 0.50 & & 1.80 \\
\hline & \multirow{3}{*}{ Autumn } & & $0-20$ & 6.40 & 1.80 & \multirow{3}{*}{0.13} & 0.20 & 6.60 & 2.90 & \multirow{3}{*}{0.19} & 2.40 \\
\hline & & & $20-40$ & 7.00 & 1.80 & & 1.80 & 6.80 & 1.80 & & 2.20 \\
\hline & & & $40-60$ & 7.60 & 1.00 & & 1.40 & 7.00 & 1.00 & & 1.80 \\
\hline & \multirow{3}{*}{ Spring } & \multirow{6}{*}{ 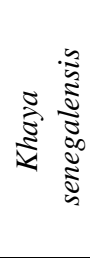 } & $0-20$ & 6.60 & 2.10 & \multirow{3}{*}{0.21} & 1.40 & 6.00 & 2.20 & \multirow{3}{*}{1.80} & 3.40 \\
\hline & & & $20-40$ & 6.60 & 1.90 & & 1.30 & 5.60 & 2.00 & & 3.20 \\
\hline & & & $40-60$ & 6.90 & 1.70 & & 1.20 & 5.40 & 0.80 & & 3.00 \\
\hline & \multirow{3}{*}{ Autumn } & & $0-20$ & 6.60 & 2.00 & \multirow{3}{*}{0.13} & 2.40 & 7.00 & 2.30 & \multirow{3}{*}{0.19} & 1.80 \\
\hline & & & $20-40$ & 6.90 & 1.10 & & 2.20 & 7.00 & 2.20 & & 1.40 \\
\hline & & & $40-60$ & 7.00 & 1.60 & & 1.80 & 7.20 & 0.50 & & 1.60 \\
\hline & \multirow{3}{*}{ Spring } & \multirow{9}{*}{ 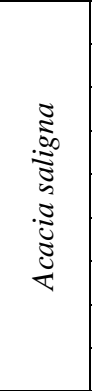 } & $0-20$ & 6.30 & 2.00 & \multirow{3}{*}{0.21} & 2.00 & 6.70 & 2.00 & \multirow{3}{*}{1.80} & 3.00 \\
\hline & & & $20-40$ & 6.90 & 1.70 & & 1.70 & 6.70 & 1.80 & & 2.80 \\
\hline & & & $40-60$ & 7.00 & 1.70 & & 1.70 & 6.60 & 0.90 & & 2.40 \\
\hline & \multirow{3}{*}{ Autumn } & & $0-20$ & 6.60 & 5.60 & \multirow{3}{*}{0.13} & 1.90 & 6.90 & 2.20 & \multirow{3}{*}{0.19} & 2.00 \\
\hline & & & $20-40$ & 5.60 & 5.60 & & 1.90 & 6.90 & 2.00 & & 2.20 \\
\hline & & & $40-60$ & 5.40 & 5.40 & & 1.90 & 6.70 & 0.90 & & 2.00 \\
\hline \multirow{3}{*}{ 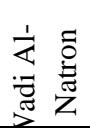 } & & & $0-20$ & 7.60 & 1.40 & & 4.50 & 7.60 & 1.50 & & 4.60 \\
\hline & Spring & & $20-40$ & 7.54 & 1.10 & 1.53 & 4.22 & 7.50 & 1.10 & 1.50 & 4.20 \\
\hline & & & $40-60$ & 7.04 & 1.10 & & 3.55 & 7.00 & 1.10 & & 3.50 \\
\hline & & & $0-20$ & 6.60 & 2.30 & & 2.30 & 6.00 & 1.80 & & 2.00 \\
\hline & Autumn & .0 & $20-40$ & 6.90 & 2.10 & 0.21 & 2.10 & 5.60 & 1.80 & 1.80 & 1.90 \\
\hline שֶ & & $y$ & $40-60$ & 7.20 & 1.20 & & 1.20 & 5.60 & 0.90 & & 3.00 \\
\hline శ్ & & $\bar{\Xi}$ & $0-20$ & 7.00 & 1.90 & & 1.90 & 6.00 & 2.00 & & 2.40 \\
\hline$\pi$ & Spring & 2 & $20-40$ & 7.40 & 1.90 & 0.13 & 1.60 & 6.00 & 1.90 & 0.19 & 3.80 \\
\hline & & & $40-60$ & 7.60 & 1.30 & & 1.30 & 6.00 & 0.90 & & 1.60 \\
\hline
\end{tabular}

Fayoum J. Agric. Res. \& Dev., Vol.25, No.1, January, 2011 
Table (5): Heavy metal concentrations (means \pm Sd) in cultivated soil at both studied areas during the tested periods.

\begin{tabular}{|c|c|c|c|c|c|c|}
\hline \multirow{2}{*}{ Sites } & \multirow{2}{*}{ Tree species } & \multirow{2}{*}{$\begin{array}{c}\text { Depth } \\
(\mathrm{cm})\end{array}$} & \multicolumn{4}{|c|}{ Heavy metal concentrations ( $\mathrm{mg} \mathrm{kg}^{-1}$ soil) } \\
\hline & & & $\mathrm{Cd}$ & Co & $\mathrm{Ni}$ & $\mathrm{Pb}$ \\
\hline \multirow{9}{*}{$\begin{array}{l}\text { Sadat } \\
\text { City }\end{array}$} & \multirow{3}{*}{ Morus alba } & $0-20$ & $0.704 \pm 1.104$ & $0.411 \pm 0.366$ & $8.315 \pm 9.309$ & $5.7747 \pm 8.440$ \\
\hline & & $20-40$ & $0.001 \pm 0.001$ & $0.031 \pm 0.032$ & $1.786 \pm 1.901$ & $0.722 \pm 0.532$ \\
\hline & & $40-60$ & $0.083 \pm 0.022$ & $0.076 \pm 0.011$ & $11.323 \pm 7.623$ & $4.861 \pm 7.496$ \\
\hline & \multirow{3}{*}{$\begin{array}{c}\text { Khaya } \\
\text { senegalensis }\end{array}$} & $0-20$ & $0.089 \pm 0.152$ & $0.173 \pm 0.233$ & $3.617 \pm 2.620$ & $4.434 \pm 7.605$ \\
\hline & & $20-40$ & $0.012 \pm 0.016$ & $0.036 \pm 0.041$ & $6.379 \pm 6.842$ & $1.694 \pm 2.417$ \\
\hline & & $40-60$ & $0.002 \pm 0.002$ & $0.024 \pm 0.031$ & $6.821 \pm 5.326$ & $0.170 \pm 0.080$ \\
\hline & \multirow{6}{*}{ Acacia saligna } & $0-20$ & $0.089 \pm 0.152$ & $0.173 \pm 0.233$ & $4.445 \pm 2.842$ & $4.434 \pm 7.605$ \\
\hline & & $20-40$ & $0.160 \pm 0.275$ & $0.098 \pm 0.098$ & $6.379 \pm 6.842$ & $1.694 \pm 2.417$ \\
\hline & & $40-60$ & $0.057 \pm 0.002$ & $0.098 \pm 0.113$ & $6.821 \pm 5.326$ & $2.197 \pm 3.596$ \\
\hline \multirow{3}{*}{$\begin{array}{c}\text { Wadi } \\
\text { Al- } \\
\text { Natron }\end{array}$} & & $0-20$ & 0.000 & 0.058 & 1.109 & 0.175 \\
\hline & & $20-40$ & 0.000 & 0.125 & 1.633 & 0.083 \\
\hline & & $40-60$ & 0.000 & 0.128 & 2.528 & 0.000 \\
\hline \multirow{3}{*}{$\begin{array}{l}\text { Sadat } \\
\text { City }\end{array}$} & \multirow{3}{*}{ Populus nigra } & $0-20$ & $0.012 \pm 0.019$ & $0.185 \pm 0.267$ & $8.310 \pm 14.283$ & $6.853 \pm 11.936$ \\
\hline & & $20-40$ & $0.002 \pm 0.003$ & $0.023 \pm 0.027$ & $1.160 \pm 1.713$ & $3.151 \pm 5.429$ \\
\hline & & $40-60$ & $0.051 \pm 0.089$ & $0.112 \pm 0.157$ & $6.334 \pm 9.796$ & $3.087 \pm 5.257$ \\
\hline
\end{tabular}

\section{RESULTS:}

I. Statistically relationships between heavy metal concentrations, atmospheric temperature, irrigation water salinity and soil $\mathrm{pH}$ :

a. Atmospheric temperature:

Table (6) reveals a negative correlation between $\mathrm{Cd}$, $\mathrm{Co}$ and $\mathrm{Pb}$ concentrations in roots of Acacia, Khaya and Populus, and atmospheric temperature. On the other hand, it reveals a positive correlation with $\mathrm{Ni}$ concentration in root of Khaya and all metal concentrations in Morus root and atmospheric temperature.

Table (6): Simple correlation coefficients between atmospheric temperature and heavy metal concentrations in root of the tree species during studied periods.

\begin{tabular}{|c|c|c|c|c|}
\hline \multirow{2}{*}{$\begin{array}{c}\text { Heavy } \\
\text { Metal }\end{array}$} & \multicolumn{4}{|c|}{ Tree species } \\
\cline { 2 - 5 } & Morus alba & Acacia saligna & Khaya senegalensis & Populus nigra \\
\hline $\mathrm{Cd}$ & 0.597 & -0.276 & -0.010 & -0.281 \\
\hline $\mathrm{Co}$ & 0.519 & -0.277 & -0.343 & -0.287 \\
\hline $\mathrm{Ni}$ & 0.078 & -0.058 & 0.108 & -0.234 \\
\hline $\mathrm{Pb}$ & 0.734 & -0.284 & -0.248 & -0.169 \\
\hline
\end{tabular}

\section{b. Irrigation water salinity:}

Table (7) reveals that there is a negative correlation between soil metal concentrations under Khaya and Populus and the irrigation water salinity, while soil supporting Morus shows a negative correlation between water salinity and its $\mathrm{Pb}$ concentrations only. On the other hand, soil supporting Acacia at the first area shows a negative correlation between soil $\mathrm{Pb}$ and $\mathrm{Co}$ concentrations and irrigation water salinity. 
Table (7): Simple correlation coefficients between irrigation water salinity (EC) and heavy metal concentrations in soil supporting the tree species during studied periods.

\begin{tabular}{|c|c|c|c|c|}
\hline \multirow{2}{*}{$\begin{array}{l}\text { Heavy } \\
\text { Metal }\end{array}$} & \multicolumn{4}{|c|}{ Tree species } \\
\hline & Morus alba & Acacia saligna & Khaya senegalensis & Populus nigra \\
\hline $\mathrm{Cd}$ & 0.545 & 0.070 & -0.130 & -0.180 \\
\hline $\mathrm{Co}$ & 0.390 & -0.394 & -0.396 & -0.192 \\
\hline $\mathrm{Ni}$ & 0.298 & 0.294 & -0.141 & -0.060 \\
\hline $\mathrm{Pb}$ & -0.047 & -0.086 & -0.119 & -0.120 \\
\hline
\end{tabular}

Morus metal root concentration shows a positive correlation with irrigation water salinity, where Acacia $\mathrm{Ni}$ root concentration, Khaya $\mathrm{Cd}$ and $\mathrm{Ni}$ root concentration and Populus $\mathrm{Pb}$ - root concentration show a positive correlation with irrigation water salinity (Table 8).

Table (8): Simple correlation coefficients between irrigation water salinity (EC) and heavy metal concentrations in root of the tree species during studied periods.

\begin{tabular}{|c|c|c|c|c|}
\hline \multirow{2}{*}{$\begin{array}{c}\text { Heavy } \\
\text { Metal }\end{array}$} & \multicolumn{4}{|c|}{ Tree species } \\
\cline { 2 - 5 } & Morus alba & Acacia saligna & Khaya senegalensis & Populus nigra \\
\hline $\mathrm{Cd}$ & 0.128 & -0.106 & 0.100 & -0.110 \\
\hline $\mathrm{Co}$ & 0.612 & -0.103 & -0.168 & -0.116 \\
\hline $\mathrm{Ni}$ & 0.286 & 0.088 & 0.307 & -0.062 \\
\hline $\mathrm{Pb}$ & 0.826 & -0.183 & -0.078 & 0.004 \\
\hline
\end{tabular}

\section{c. Soil pH:}

Table (9) reveals a positive correlation between soil $\mathrm{pH}$ at $40-60 \mathrm{~cm}$ depth and Morus root Co concentration, positive correlation with $\mathrm{Cd}, \mathrm{Co}, \mathrm{Ni}$ and $\mathrm{Pb}$ concentration in Acacia root and negative correlation between Khaya and Populus root metal concentrations and soil $\mathrm{pH}$.

Table (9): Simple correlation coefficients between $\mathrm{pH}$ values at $40-60 \mathrm{~cm}$ depth and heavy metal concentrations in root of the tree species during studied periods.

\begin{tabular}{|c|c|c|c|c|}
\hline \multirow{2}{*}{$\begin{array}{c}\text { Heavy } \\
\text { Metal }\end{array}$} & \multicolumn{4}{|c|}{ Tree species } \\
\cline { 2 - 5 } & Morus alba & Acacia saligna & Khaya senegalensis & Populus nigra \\
\hline $\mathrm{Cd}$ & -0.456 & 0.170 & -0.930 & -0.703 \\
\hline $\mathrm{Co}$ & 0.519 & 0.143 & -0.990 & -0.660 \\
\hline $\mathrm{Ni}$ & -0.399 & 0.151 & -0.838 & -0.660 \\
\hline $\mathrm{Pb}$ & -0.260 & 0.165 & -0.981 & -0.732 \\
\hline
\end{tabular}

II. Heavy metal contents in oxidation pond and natural underground waters:

The concentration of heavy metals $(\mathrm{Cd}, \mathrm{Co}, \mathrm{Ni}$ and $\mathrm{Pb})$ in irrigation water samples collected from the oxidation pond and natural underground water, Table 3 , showed an always seasonal difference as regards different elements in the oxidation pond water $(\mathrm{P}<0.001)$. Generally metal concentrations were noticeably higher during 2010 than 2009. Also, concentrations of $\mathrm{Cd}, \mathrm{Co}, \mathrm{Ni}$ and $\mathrm{Pb}$, as mean values during autumn 2010 at Wadi Al-Natron area, were 0.003, 0.076, 0.989 and 0.007

Fayoum J. Agric. Res. \& Dev., Vol.25, No.1, January, 2011 
$\mathrm{mg} \mathrm{L}{ }^{-1}$, respectively. Moreover, nickel concentrations at the same area was recorded the highest value, which exceeded the FAO critical limit of fresh water.

III. Heavy metal concentrations in the studied soil:

a. Available cadmium $(\boldsymbol{C d})$ :

Regarding soil supporting Acacia at Sadat City area as compared to Wadi AlNatron area, Table 5, the mean values of $\mathrm{Cd}$ were zero in all soil depths. Available Cd concentration on the surface layer $(0-20 \mathrm{~cm})$ of soil supporting Morus showed the highest concentration where $(\mathrm{P}<0.05)$, while in the subsurface layer $(20-40 \mathrm{~cm})$ soil supporting Acacia showed the highest significant $\mathrm{Cd}$ concentration $(\mathrm{P}<0.05)$ and at the deep layer $(40-60 \mathrm{~cm})$ showed insignificant differences between soil supporting different species $(\mathrm{P}>0.05)$.

\section{b. Available cobalt (Co):}

Concerning soil supporting Acacia at Sadat City area as compared to Wadi Al-Natron area, Table 5, the mean values of Co were $0.058,0.125$ and $0.128 \mathrm{mg}$ $\mathrm{kg}^{-1}$ on the surface $0-20 \mathrm{~cm}$, subsurface $20-40 \mathrm{~cm}$ and deep $40-60 \mathrm{~cm}$ layers, respectively. Statistically, there was insignificant differences for Co concentrations in soil supporting different species $(\mathrm{P}>0.05)$ at the surface $0-20 \mathrm{~cm}$ layer. Also, Co concentrations showed the highest significant Co values $(\mathrm{P}<0.05)$ in the surface 0 $20 \mathrm{~cm}$ layer soil supporting Acacia, while there was insignificant difference between soil supporting different species $(\mathrm{P}>0.05)$ at the deep 40-60 layer.

\section{c. Available nickel (Ni):}

With respect to soil supporting Acacia at Sadat City area as compared to Wadi Al-Natron area, Table 5, Ni contents were $1.109,1.633$ and $2.528 \mathrm{mg} \mathrm{kg}^{-1}$ for the surface $0-20 \mathrm{~cm}$, subsurface $20-40 \mathrm{~cm}$ and deep $40-60 \mathrm{~cm}$ layers, respectively.

Also, there was insignificantly difference between Ni concentrations in soil supporting the different tree species $(\mathrm{P}>0.05)$ at the surface layer $(0-20 \mathrm{~cm})$. as for the subsurface layer $(20-40 \mathrm{~cm})$ of soil supporting Acacia and Khaya, the statistical analysis showed a highly significant difference in Ni concentrations $(\mathrm{P}<0.05)$, while at the deep layer $(40-60 \mathrm{~cm})$ there was an insignificant difference between soil supporting the different tree species $(\mathrm{P}>0.05)$.

\section{d. Available lead $(\mathrm{Pb})$ :}

Regarding soil supporting Acacia at Sadat City area as compared to Wadi AlNatron area, Table 5, the mean values of $\mathrm{Pb}$ were $0.175,0.083$ and $0.000 \mathrm{mg} \mathrm{kg}^{-1}$ for the surface $(0-20 \mathrm{~cm})$, subsurface $(20-40 \mathrm{~cm})$ and deep $(40-60 \mathrm{~cm})$, respectively. All soil depths supporting the different tree species showed insignificant difference between $\mathrm{Pb}$ concentrations in soil supporting various tree species $(\mathrm{P}>0.05)$,

$I V$. Heavy metal accumulations in the different plant organs (root, stem, leaf and fruit) of the investigated tree species:

\section{a. Cadmium concentration:}

Table (10) showed that Cd concentration as mean values in Acacia cultivated at Wadi Al-Natron were $0.001,0.002$ and $00.000 \mathrm{mg} \mathrm{kg}^{-1}$ in tree roots, stems and leaves. At Sadat City area, the root Cd-concentration mean values in both Acacia and Khaya were similarly low, in contrast Morus and Populus have similarly the highest $\mathrm{Cd}$ concentration in their roots during studied period $(\mathrm{P}<0.05)$. There were insignificant differences in Cd-concentrations of tree stems, leaves and fruits of various tree species $(\mathrm{P}>0.05)$ 
MONITORING OF ENVIRONMENTAL POLLUTION IN.

Table (10): Heavy metal concentrations (means \pm Sd) in plant organs of the studied tree species during the tested periods.

\begin{tabular}{|c|c|c|c|c|c|c|}
\hline \multirow{2}{*}{ Area } & \multirow{2}{*}{ Tree species } & \multirow{2}{*}{$\begin{array}{l}\text { Plant } \\
\text { organ }\end{array}$} & \multicolumn{4}{|c|}{ Heavy metal concentrations $\left(\mathrm{mg} \mathrm{kg}^{-1}\right)$} \\
\hline & & & $\mathrm{Cd}$ & $\mathrm{Co}$ & $\mathrm{Ni}$ & $\mathrm{Pb}$ \\
\hline \multirow{11}{*}{$\begin{array}{l}\text { Sadat } \\
\text { City }\end{array}$} & \multirow{4}{*}{ Morus alba } & Root & $0.149 \pm 0.1025$ & $0.481 \pm 0.528$ & $1.950 \pm 1.901$ & $5.708 \pm 3.540$ \\
\hline & & Stem & $0.117 \pm 0.078$ & $0.123 \pm 0.129$ & $1.782 \pm 1.065$ & $1.105 \pm 1.410$ \\
\hline & & Leaf & $0.039 \pm 0.065$ & $0.377 \pm 0.673$ & $0.377 \pm 0.673$ & $12.832 \pm 23.144$ \\
\hline & & Fruit & $0.035 \pm 0.064$ & $0.373 \pm 0.676$ & $2.755 \pm 4.985$ & $3.243 \pm 5.866$ \\
\hline & \multirow{4}{*}{$\begin{array}{c}\text { Khaya } \\
\text { senegalensis }\end{array}$} & Root & $0.009 \pm 0.010$ & $0.013 \pm 0.020$ & $0.440 \pm 0.374$ & $0.543 \pm 0.860$ \\
\hline & & Stem & $0.049 \pm 0.084$ & $0.046 \pm 0.078$ & $4.804 \pm 8.061$ & $0.874 \pm 1.410$ \\
\hline & & Leaf & $0.056 \pm 0.102$ & $0.046 \pm 0.078$ & $0.046 \pm 0.078$ & $0.419 \pm 0.751$ \\
\hline & & Fruit & $\mathrm{ns}$ & $\mathrm{ns}$ & $\mathrm{ns}$ & $\mathrm{Ns}$ \\
\hline & \multirow{8}{*}{$\begin{array}{l}\text { Acacia } \\
\text { saligna }\end{array}$} & Root & $0.031 \pm 0.055$ & $0.066 \pm 0.112$ & $0.750 \pm 0.870$ & $2.255 \pm 4.034$ \\
\hline & & Stem & $0.025 \pm 0.045$ & $0.031 \pm 0.052$ & $1.014 \pm 1.019$ & $4.677 \pm 8.126$ \\
\hline & & Leaf & $0.166 \pm 0.300$ & $0.042 \pm 0.044$ & $0.042 \pm 0.044$ & $0.602 \pm 1.081$ \\
\hline \multirow{5}{*}{$\begin{array}{c}\text { Wadi } \\
\text { Al- } \\
\text { Natron }\end{array}$} & & Fruit & $0.049 \pm 0.089$ & $0.429 \pm 0.776$ & $0.352 \pm 0.636$ & $0.832 \pm 1.505$ \\
\hline & & Root & 0.001 & 0.000 & 0.601 & 0.040 \\
\hline & & Stem & 0.002 & 0.000 & 0.604 & 0.049 \\
\hline & & Leaf & 0.000 & 0.000 & 0.447 & 0.094 \\
\hline & & Fruit & ns & ns & ns & $\mathrm{Ns}$ \\
\hline \multirow{4}{*}{$\begin{array}{l}\text { Sadat } \\
\text { City }\end{array}$} & \multirow{4}{*}{ Populus nigra } & Root & $0.168 \pm 0.298$ & $0.119 \pm 0.214$ & $1.181 \pm 1.851$ & $0.361 \pm 0.480$ \\
\hline & & Stem & $0.181 \pm 0.264$ & $0.634 \pm 0.823$ & $8.258 \pm 12.795$ & $4.049 \pm 6.606$ \\
\hline & & Leaf & $0.011 \pm 0.019$ & $0.015 \pm 0.019$ & $0.015 \pm 0.019$ & $0.011 \pm 0.019$ \\
\hline & & Fruit & ns & ns & ns & $\mathrm{Ns}$ \\
\hline
\end{tabular}

\section{b. Cobalt concentration:}

The Co concentration as mean values in Acacia cultivated at Wadi AlNatron, Table 10, were zero in all plant organs. Root

Co concentration of Morus at Sadat City area showed the highest concentration, meanwhile its soil has no the lowest value. On the other hand, Acacia showed the highest Co contents in the subsurface soil depth $(20-40 \mathrm{~cm})$, while its roots have no the lowest concentration $(\mathrm{P}<0.001)$. Stem-Co concentrations were showed significant differences between various tree species $(\mathrm{P}$ $<0.05)$. Co-concentration as mean values in tree stems could be categorized in an ascending order of Populus > Morus > Khaya > Acacia. Leaf-Co concentrations showed wide variations in the investigated tree species $(\mathrm{P}<0.05)$. Its concentrations could be categorized in an ascending order of Morus > Khaya > Acacia > Populus. Fruit Co concentrations showed insignificant differences between the investigated tree species $(\mathrm{P}>0.05)$.

\section{c. Nickel concentration:}

Nickel concentrations as mean values in Acacia cultivated at Wadi AlNatron, Table 10, were recorded $0.601,0.604$ and $0.447 \mathrm{mg} \mathrm{kg}^{-1}$ in the tree roots, stems and leaves, respectively. The highest values of Ni concentration were found in the subsurface layer $(20-40 \mathrm{~cm})$ of soil supporting both Acacia and Khaya of Sadat City, with insignificant differences in their root concentrations $(\mathrm{P}>0.05)$. There were also no differences in Ni stem concentration between various species $(\mathrm{P}$ $>0.05)$. Meanwhile, there were significant differences between tree species for leaves $(\mathrm{P}<0.05)$, where $\mathrm{Ni}$ concentrations could be arranged in an ascending order of Morus > Khaya > Acacia > Populus. As for Ni concentration in fruits, There were insignificant differences between various tree species $(\mathrm{P}>0.05)$.

\section{d. Lead concentration:}

The obtained data of $\mathrm{Pb}$ concentration, Table 10, revealed that its mean values in Acacia cultivated at Wadi Al-Natrons area during autumn 2010 were

Fayoum J. Agric. Res. \& Dev., Vol.25, No.1, January, 2011 
$0.040,0.049$ and $0.094 \mathrm{mg} \mathrm{kg}^{-1}$ in tree roots, stems and leaves. Morus root seems to absorb $\mathrm{Pb}$ more than other organs of tree species $(\mathrm{P}<0.001)$. In tree stems, there was insignificant differences between the investigated tree species, $(\mathrm{P}>0.05)$. While, leaf $\mathrm{Pb}$ concentrations exhibited a wide variation between the investigated tree species $(\mathrm{P}<0.05)$, with an ascending order of Morus > Acacia > Khaya > Populus. Whereas, there was insignificant difference between tree species for fruits $(\mathrm{P}>0.05)$

$V$. Sequestration preferences and translocation factors:

The data of different metal sequestration preferences within plant organs in various investigated tree species are presented in Table 11. Morus root seems to sequestrate $\mathrm{Cd}, \mathrm{Co}$ and $\mathrm{Ni}$, while $\mathrm{Pb}$ found to be sequestrated more in leaves. Khaya stem seems to sequestrate $\mathrm{Ni}$ and $\mathrm{Pb}$, while $\mathrm{Co}$ found to be similarly sequestrated in stems and leaves.

Table (11): Metal sequestration preferences within plant organs and tree species during studied period.

\begin{tabular}{|c|c|c|c|c|}
\hline Tree species & $\mathrm{Cd}$ & $\mathrm{Co}$ & $\mathrm{Ni}$ & $\mathrm{Pb}$ \\
\hline Morus alba & $\mathrm{R}>\mathrm{S}>\mathrm{L}$ & $\mathrm{R}>\mathrm{L}>\mathrm{S}$ & $\mathrm{R}>\mathrm{S}>\mathrm{L}$ & $\mathrm{L}>\mathrm{R}>\mathrm{S}$ \\
\hline Khaya sengalensis & $\mathrm{L}>\mathrm{S}>\mathrm{R}$ & $\mathrm{S}=\mathrm{L}>\mathrm{R}$ & $\mathrm{S}>\mathrm{L}>\mathrm{R}$ & $\mathrm{S}>\mathrm{L}>\mathrm{R}$ \\
\hline Acacia saligna & $\mathrm{L}>\mathrm{R}>\mathrm{S}$ & $\mathrm{R}>\mathrm{L}>\mathrm{S}$ & $\mathrm{S}>\mathrm{R}>\mathrm{L}$ & $\mathrm{L}>\mathrm{R}>\mathrm{S}$ \\
\hline Populus nigra & $\mathrm{R}>\mathrm{S}>\mathrm{L}$ & $\mathrm{S}>\mathrm{R}>\mathrm{L}$ & $\mathrm{S}>\mathrm{R}>\mathrm{L}$ & $\mathrm{S}>\mathrm{R}>\mathrm{L}$ \\
\hline
\end{tabular}

$\mathrm{R}=$ Root $\mathrm{S}=$ Stem $\quad \mathrm{L}=$ Leaf

Metal sequestration preferences following different order within tree organs of Acacia. Populus stem seems to sequestrate $\mathrm{Co}, \mathrm{Ni}$ and $\mathrm{Pb}$, while $\mathrm{Cd}$ seems to be sequestrated more in Populus roots.

\section{Metal translocation factors:}

Data showed that all investigated tree species are able to translocated metals from roots to shoots, where all values of translocation factors are above $1 . \mathrm{Cd}, \mathrm{Co}$, $\mathrm{Ni}$ and $\mathrm{Pb}$ translocation factor values in Morus are 1.00, 1.03, 1.10 and 2.44, respectively. The corresponding translocation factor values are 4.50, 1.10, 1.40 and 2.29 in Khaya vs 11.66, 2.46, 11.01 and 2.38 in Acacia and 1.14, 3.18, 1.18 and 11.84 in Populus, respectively.

VII. Relation between metal translocation factors in the tree species and recorded atmospheric temperature in different studied seasons:

The obtained data in Table 12 showed that Morus Co and Ni translocation factors were the highest values at the highest temperature. In contrast, an opposite relation shows for Populus $\mathrm{Cd}, \mathrm{Co}, \mathrm{Ni}$ and $\mathrm{Pb}$ translocation factors, where the highest values were recorded at the lowest temperature. 
MONITORING OF ENVIRONMENTAL POLLUTION IN..........

Table (12): Translocation factors for tree species as affected by atmospheric temperature.

\begin{tabular}{|c|c|c|c|c|c|}
\hline Tree species & Temperature & $\mathrm{Cd}$ & $\mathrm{Co}$ & $\mathrm{Ni}$ & $\mathrm{Pb}$ \\
\hline \multirow{4}{*}{\begin{tabular}{c} 
Morus alba \\
\cline { 2 - 6 }
\end{tabular}} & 9.26 & 0.000 & 0.50 & 1.533 & 1.800 \\
\cline { 2 - 6 } & 23.33 & 0.000 & 0.50 & 0.694 & 0.232 \\
\cline { 2 - 6 } & 15.20 & 0.513 & 0.76 & 3.349 & 6.401 \\
\hline \multirow{3}{*}{$\begin{array}{c}\text { Populus } \\
\text { nigra }\end{array}$} & 26.30 & 0.563 & 48.00 & 10.670 & 0.191 \\
\cline { 2 - 6 } & 23.33 & 121.00 & 606.00 & 300.560 & 254.000 \\
\cline { 2 - 6 } & 15.20 & 1.00 & 0.00 & 21.196 & 1.628 \\
\hline \multirow{4}{*}{$\begin{array}{c}\text { Acacia } \\
\text { saligna }\end{array}$} & 26.30 & 0.40 & 0.00 & 3.741 & 15.190 \\
\cline { 2 - 6 } & 9.26 & 0.00 & 0.500 & 0.977 & 1.000 \\
\cline { 2 - 6 } & 23.33 & 0.00 & 0.766 & 9.000 & 15.105 \\
\hline \multirow{3}{*}{$\begin{array}{c}\text { Khaya } \\
\text { senegalensis }\end{array}$} & 15.20 & 6.18 & 0.500 & 2.030 & 1.628 \\
\cline { 2 - 6 } & 26.30 & 0.50 & 48.000 & 1.203 & 1.280 \\
\cline { 2 - 6 } & 23.26 & 0.000 & 0.666 & 1.071 & 0.666 \\
\cline { 2 - 6 } & 15.20 & 0.555 & 3.333 & 0.900 & 0.225 \\
\hline
\end{tabular}

Meanwhile, Acacia metal translocation factors showed different behaviour towards the various studied metals, the highest Co translocation factor showed the highest value at the highest temperature, while the reverse was true for that shows its highest translocation factor at the lowest temperature. On the other hand, there was no a specific trend between temperature and Khaya metal translocation factors. The translocation preference patterns were varied according the nature of the investigated tree species, i.e., $\mathrm{Pb}>\mathrm{Ni}>\mathrm{Co}>\mathrm{Cd}, \mathrm{Cd}>\mathrm{Pb}>\mathrm{Ni}>\mathrm{Co}, \mathrm{Cd}>\mathrm{Ni}>\mathrm{Co}>\mathrm{Pb}$ and $\mathrm{Pb}>\mathrm{Co}>\mathrm{Ni}>\mathrm{Cd}$ in Morus, Khaya, Acacia and Populus, respectively.

\section{DISCUSSION:}

a. Atmospheric temperature:

From the previous results it could be observes that there was a positive correlation between metal-root concentrations and atmospheric temperature. These findings are in agreement with Helal et al. (1997) who found that plants grown at $25^{\circ} \mathrm{C}$ accumulate higher levels of $\mathrm{Cd}, \mathrm{Ni}$ and $\mathrm{Pb}$ than those grown at $15^{\circ} \mathrm{C}$; this appears to be caused by a progressive decline in $\mathrm{pH}$ and organic matter soil status.

\section{b. Evaluation of water samples:}

The variations in apparent correlation between soil metals and salinity values (slight to moderate) might not be attributed to the irrigation source, but to other factors such as fertilizers, fungicides and bactericides. These opinions are more agreement with the findings outlined by Abo El-Roos et al. (1996) who reported irrigation water salinity affects heavy metals availability to plants, where high salinity causes an increase in their concentrations. The negative correlation between salinity and metal concentrations may be due to different tree root behavior in the rhizospher, soil properties and pollution with other specific ions of heavy metals. The highest degree of hardness may be due to the presence of gypsum and limestone sediments that are rich in calcium (Said, 2002)

c. Metal ions in water:

The maximum concentration of $\mathrm{Cd}, \mathrm{Co}$ and $\mathrm{Pb}$ in the oxidation pond samples which observed during autumn 2010 and highest value of Ni observed in 2010 spring season may be due to the increasing industrial activities during this periods $(\mathrm{P}<0.001)$.

Fayoum J. Agric. Res. \& Dev., Vol.25, No.1, January, 2011 
Statistical analysis shows significant differences between periodically seasons in water $\mathrm{Cd}$ contents. Concerning high Co and Ni concentrations observed during 2010 autumn season in the natural underground water at Wadi Al-Natron area may be associated with natural process of chemical weathering of sedimentary rocks, soil leaching of metal polluted water is an important environmental factor as well as other industrial activities in such region. There is always seasonal difference as regards different elements in wastewater, during 2010 the metal contents is noticeably higher in 2010 than 2009, may be due to the different year industrial activities.

\section{d. Soil characteristics:}

Soil $\mathrm{pH}$ values at a depth $40-60 \mathrm{~cm}$ in soil supporting Morus has a negative significant correlation with heavy metal concentrations in plant roots, except with Co that shows a positive correlation with soil $\mathrm{pH}$, may be due to different root interactions in the rhizospher, soil properties and other Co-metal pollution interactions in soil. While, a negative correlation with soil $\mathrm{pH}$ may be due to tree root behavior with metal uptake in the rhizospher and rhizospheric interactions that may led to modification of root zone $\mathrm{pH}$ value with different metals and tree species. That was true, since plant roots have been supported to contribute with the mobilization of nutrients in some species (Huang, 1997).

The highest $\mathrm{Cd}$ concentration is observed in the surface layer $(0-20 \mathrm{~cm})$ of soil supporting Morus during autumn 2010, this may be due to the higher soil organic matter in the surface layer because of plant litter accumulation which reduces $\mathrm{Cd}$ mobility, and consequently increasing its concentration in the upper layer of the soil profile.

Concerning the highest significantly $\mathrm{Cd}$ concentration that is observed in the subsurface $20-40 \mathrm{~cm}$ layer of soil supporting Acacia at Sadat City area, may be attributed to the relatively high soil $\mathrm{pH}$ value that reduces $\mathrm{Cd}$ mobility, and consequently increases its concentration in such soil depth. The widely variations in $\mathrm{Cd}$ values in the studied soil may be due to different $\mathrm{pH}$ values in various soil depths. The highest Ni concentrations in soil supporting Acacia at Wadi Al-Natron area at the deep $40-60 \mathrm{~cm}$ layer during autumn may be due to high organic matter content that reduces metal mobility and increase its concentrations in the soil. Statistically, there was insignificant difference in $\mathrm{Ni}$ concentrations between soils supporting different tree species in the surface and deep layers $(\mathrm{P}>0.05)$, while the highest significant $\mathrm{Ni}$ concentrations observed in subsurface layer $(20-40 \mathrm{~cm})$ of soils supporting Acacia and Khaya $(\mathrm{P}<0.05)$. These results may be due to the differences in soil $\mathrm{pH}$ values and the occurrence of relatively high organic matter content that reduces the mobility of some metals. The highest $\mathrm{Pb}$ concentration in soil supporting Acacia at Wadi Al-Natron area was observed in surface $0-20 \mathrm{~cm}$ layer during autumn, may be due to the presence of plant litter on the surface layer that reduces metal mobility and increases its concentrations in the soil.

e. Heavy metal concentrations in the investigated tree roots and shoot systems.

Cd concentrations in Populus were higher in tree root $\left(0.663 \mathrm{mg} \mathrm{kg}^{-1}\right)$ than that in tree stem $\left(0.613 \mathrm{mg} \mathrm{kg}^{-1}\right)$ during spring and the lowest value of $\mathrm{Cd}$ in the Populus organs observed in the new leaves $\left(0.042 \mathrm{mg} \mathrm{kg}^{-1}\right)$ after 2009 winter dormant season. While, the highest concentration of $\mathrm{Cd}$ in Morus root was associated with high concentration in surface $0-20 \mathrm{~cm}$ layer, may be due to the upward migration of soluble metal and slow translocation rate from root to shoot with higher organic matter values in the surface layer. Acacia and Khaya trees have similar affinity for low Cd root absorb, because of the remarkable degree of uptake of specific metal as well as the mechanism of metal selectivity (Still et al., 1980).

Fayoum J. Agric. Res. \& Dev., Vol.25, No.1, January, 2011 
Statistical analysis of Co concentration in tree root of the investigated tree species revealed that Co highest concentration was observed in Morus root, but its value in soil supporting Morus has not the lowest concentration. In contrast, Acacia root has the lowest Co concentration and the highest concentration observed in the subsurface layer $(20-40 \mathrm{~cm})$ of soil supporting Acacia tree. The highest Co concentration was observed in Morus root, while it in supporting soil has not the lowest concentration in the subsurface layer $(20-40 \mathrm{~cm})$, may be due to the upward migration of soluble metal. These results showed a different behavior of Morus root uptake toward $\mathrm{Cd}$ and $\mathrm{Co}$, where the Co content during spring showed higher contents in leaves $\left(1.495 \mathrm{mg} \mathrm{kg}^{-1}\right)$ than that in stem $\left(0.904 \mathrm{mg} \mathrm{kg}^{-1}\right)$ and the lowest value $\left(0.353 \mathrm{mg} \mathrm{kg}^{-1}\right)$ in root. That indicates higher Co translocation rate from tree roots to stems than those of $\mathrm{Cd}$, may be due to the different response of Morus towards the essential elements such as Co than that of toxic ones such as $\mathrm{Cd}$, where for most of toxic metals the rate of metal translocation from roots to stems that was much lower compared with the rate of uptake of non-toxic metals (Huang and Cunningham, 1996). Co concentrations of Acacia at Sadat City area achieved 0.066 times higher in tree root, 0.031 times higher in tree stem and 0.042 times higher in tree leaves than those of the same tree cultivated in Wadi Al-Natron area, due to irrigation with wastewater.

$\mathrm{Ni}$ concentrations in various tree leaves take the following descending order: Morus > Khaya > Acacia > Populus, these may be due to different rate of $\mathrm{Ni}$ translocation from tree stem to leaves in different species, while there was insignificant difference in fruit $\mathrm{Ni}$ concentration between Morus and Acacia $(\mathrm{P}>0.05)$. Ni concentrations of Acacia at Sadat City area achieved 1.247 times higher in tree root, 1.678 times higher in tree stem and 0.093 times higher in tree leaves than those of the same tree cultivated in Wadi Al-Natron, due to irrigation with wastewater. The highest $\mathrm{Ni}$ concentration was observed in subsurface layer $(20-40 \mathrm{~cm})$ of soil supporting both Acacia and Khaya tree species, but these values in soil are not associated with any root concentration with significant differences. This may be due to the higher rate of translocation of $\mathrm{Ni}$ from tree roots to stems, where the highest concentration was appeared in tree leaves. The likely reason may be due to reduction of $\mathrm{Ni}$ concentration in tree root and stem after translocation to leaves as a result of the growth dilution effect. The highest concentration was observed in Morus leaves and fruit due to high rate of Ni translocation from stem to leaves as a result of different metal accumulation sites in different plants according to Vazques et al. (1992).

The highest Ni concentration was found in Acacia leaves at Sadat City area during autumn. It can be concluded that these results were generally high due to the high concentration of $\mathrm{Ni}$ in irrigation water of natural underground water, which absorbed by plant root, then translocated to the upper stem system, but these values still lower than those irrigated with the oxidation pond at Sadat City area. As illustrated presented in Table (11), the data revealed that the highest concentration of $\mathrm{Pb}$ observed in Morus root, and its concentration in tree root seems to absorb $\mathrm{Pb}$ more than roots of the other three species. That was true, since the $\mathrm{Pb}$ concentration in soils supporting the four tree species were similar. Morus root of $\mathrm{Pb}$ concentration gives a supportive evidence of its capability to absorb $\mathrm{Pb}$ despite as well as it was the least mobile element $(\mathrm{P}<0.001)$. Statistical analysis showed insignificant difference between $\mathrm{Pb}$ concentration in stem and fruit of tree species $(\mathrm{P}>0.05) . \mathrm{Pb}$ concentration in leaves tends to be found in different concentrations in various investigated tree species

Fayoum J. Agric. Res. \& Dev., Vol.25, No.1, January, 2011 
$(\mathrm{P}<0.05) . \mathrm{Pb}$ concentrations in leaves showed an ascending order of Morus $>$ Acacia $>$ Khaya $>$ Populus.

The ability of Morus root to absorb $\mathrm{Pb}$ may be due to increasing its solubility by root exudes, which was associated with decreasing soil $\mathrm{pH}$ in the rhizosphere (Mc.Grath, 1997). The highest $\mathrm{Pb}$ concentration observed in Morus leaves (51.215 mg $\mathrm{kg}^{-1}$ ) during spring indicates high translocation rate from root to stem. These results may also due to the presence of phytochelaties, i.e., carboxylic acid that is abundant in cells of terrestrial. It could be concluded that the Pb concentrations of Acacia at Sadat City area achieved 56.375 times higher in tree root, 93.40 times higher in tree stem and 6.404 times higher in tree leaves than those of the same tree cultivated in Wadi AlNatron area, due to irrigation with wastewater.

\section{$f$. Metal sequestration preferences and translocation factor:}

All metal translocation factors were more than 1, means that metals were successfully transported from tree roots to stems. Metal translocation preferences showed an ascending orders of $\mathrm{Pb}>\mathrm{Ni}>\mathrm{Co}>\mathrm{Cd}, \mathrm{Cd}>\mathrm{Pb}>\mathrm{Ni}>\mathrm{Co}, \mathrm{Cd}>\mathrm{Ni}>\mathrm{Co}>\mathrm{Pb}$ and $\mathrm{Pb}>\mathrm{Co}>\mathrm{Ni}>\mathrm{Cd}$. in Morus, Khaya, Acacia and Populus, respectively.

\section{g. Relation between metal translocation factors in the investigated species and recorded atmospheric temperature in different study seasons:}

Morus $\mathrm{Ni}$ and Co highest translocation factors found at the highest temperature degrees, the revere was true for Populus $\mathrm{Cd}, \mathrm{Co}, \mathrm{Ni}$ and $\mathrm{Pb}$ translocation factors that showed the highest values at the lowest temperature. Acacia metal translocation factors showed different behaviour with different metals, the highest $\mathrm{Co}$ and $\mathrm{Pb}$ translocation factors showed the highest values at the highest and lowest temperatures, respectively. On the other hand, there was no relation between temperature and Khaya metal translocation factors. It can be concluded that various tree metal translocation factors have different behaviour with atmospheric temperature. In general, it was found that metal translocation factors are not only affected by atmospheric temperature but also are affected by soil $\mathrm{pH}$, organic matter and irrigation water salinity. Such conditions may be assist naturally to accelerate soil phytoremediation processes at the warm climate of desert regions.

\section{CONCLUSIONS}

Generally, Morus and Populus both acted like Cd extractors, however, the current study shows that Populus has been able to remove Cd from the soil, while Morus acted like $\mathrm{Cd}, \mathrm{Co}, \mathrm{Ni}$ and $\mathrm{Pb}$ extractor, it is classified as un-hyperaccumulators. All metals were successfully transported from tree roots to shoots for all the investigated tree species. Metal sequestration preferences in Morus, Acacia, Khaya and Populus could be categorized in the following ascending orders of:

$\mathrm{Cd}: \mathrm{R}>\mathrm{S}>\mathrm{L}, \mathrm{L}>\mathrm{R}>\mathrm{S}, \mathrm{L}>\mathrm{S}>\mathrm{R}$ and $\mathrm{R}>\mathrm{S}>\mathrm{L}$, respectively.

Co: $\mathrm{R}>\mathrm{L}>\mathrm{S}, \mathrm{R}>\mathrm{L}>\mathrm{S}, \mathrm{S}=\mathrm{L}>$ Rand $\mathrm{S}>\mathrm{R}>\mathrm{L}$, respectively.

Ni: $R>S>L, S>R>L, S>L>R$ and $S>R>L$, respectably.

$\mathrm{Pb}: \mathrm{R}>\mathrm{S}>\mathrm{L}, \mathrm{S}>\mathrm{R}>\mathrm{L}, \mathrm{S}>\mathrm{R}>\mathrm{L}$ and $\mathrm{S}>\mathrm{R}>\mathrm{L}$, respectively.

The metal concentration is not only depending on its mechanism of uptake by plant but also on the received metal-contaminated wastewater in the soil. So, metal supplying may be limited under less received wastewater in different seasons and less polluted conditions. Also, it is noticed that Ni concentrations in soil and water of the first area (Sadat City, contaminated wastewater) exceeded the recorded values of Egypt contaminated soil and water, but it exceeds the recorded values of fresh water in the second area (Wadi Al-Natron, natural underground water).

Metal translocation preferences showed different distribution patterns in Morus; Khaya;, Acacia and Populus as illustrated in the descending orders of $\mathrm{Pb}>\mathrm{Ni}>\mathrm{Co}>\mathrm{Cd}$;

Fayoum J. Agric. Res. \& Dev., Vol.25, No.1, January, 2011 
$\mathrm{Cd}>\mathrm{Pb}>\mathrm{Ni}>\mathrm{Co} ; \mathrm{Cd}>\mathrm{Ni}>\mathrm{Co}>\mathrm{Pb}$ and $\mathrm{Pb}>\mathrm{Co}>\mathrm{Ni}>\mathrm{Cd}$, respectively. $\mathrm{Ni}$ and $\mathrm{Co}$ highest translocation factors found at the highest temperature degrees for Morus only and both concentrations are more affected by soil $\mathrm{pH}$, organic matter and irrigation water salinity, which may be assist naturally to accelerate soil phytoremediation processes at warm climate of desert regions.

\section{REFERENCES}

Abo El-Roos, S.A.; Holah and Badawy, S.H. (1996). Background levels of some heavy metals in soils of Egypt and associated corn plant. Egypt. J. of Soil Sci., 4: 83-97.

ATSDR "Agency of Toxic Substances and Disease Registry" (1999). Toxicological Profile for Cadmium.

Black, C.A.; Evans, D.D.; Ensminger, L.E.; White, J.L. and Clark, F.E. (1965). Methods of Soil Analysis. Amer. Soc. Agron. Inc., Pub., Madison, Wisconsin., USA.

Do Nacimiento, C.W.A.; Amarasiriwardena, D. and Baoshan, X. (2006). Comparison of natural organic acids and synthetic chelates at enhancing phytoextraction of metals from a multimetal contaminated soil. Environmental Pollution, 140 (1): 114-123.

Gardea, J.L.; Peralta, J.R.; De La Rosa, G. and Parsons, J.G. (2005). Phytoremediation of heavy metals and study of the metal coordination by x-ray absorption spectroscopy. Coordination Chemistry Reviews, (249) 18: 1797-1810.

Helal, H.M.; Ramadan, A: Azam, F. and Prast, R. (1997). Heavy metal uptake by Lolium italicum and root mortality as affected by water irrigation "Contaminated soils: $3^{\text {rd }}$ Inter. Conference on Biochemistry of Trace Elements, Paris, France May, 1995.1997.189-194; 8 ref.

Huang, J.W. (1997). Phytoextraction of lead from contaminated soils. In phytoremediation of soil and water contaminants. E. L. Kruger et al. (Eds). The American Society, 283-298 pp.

Huang J.W. and Cunningham, S.D. (1996). Lead phytoextraction, species variation in lead uptake and translation. New Phytol., 134:75-84.

Jackson, M.L. (1973). Soil Chemical Analysis, Prentic Hall, India Private, LTD, New Delhi.

Li, Y.; Dhankher, O.P.; Carreira, L.; Lee, D.; Chen, A.; Schroeder, J.L.; Balish, R. and Meagher, R.B. (2004). Over expression of phytochelatin synthase in Arabidopsis leads to enhanced arsenic tolerance and cadmium hypersensitivity. Plant and Cell Physiology, (45) 12: 1787-1797.

Madrid, F.; Liphadzi, M.S. and Kirkham, M.B. (2003). Heavy metal displacement in chelateirrigated soil during phytoremediation. J. of Hydrology, (272) 1: 107119.

Mc. Grath, S.P. (1997). Heavy metal uptake and chemical changes in the rhizosphere of Thlaspi caerulescens and Thlaspi ochroleucum grown in contaminated soils. Plant and Soil, 188:153-159.

Nouairi, I.; Ammar, W.B.; Youssef, N.B.; Daoud, D.B.M.; Ghorbal, M.H. and Zarrouk, M. (2006). Comparative study of cadmium effects on membrane lipid composition of Brassica juncea and Brassica napus leaves. Plant Sci., (170) 3: 511-519.

Piper, C.S. (1950). Soil and Plant Analysis. Lnter. Sci. Publishers, Inc. New York USA.

Roosens, N.; Verbruggen, N.; Meerts, P.; Ximenez-Embun, P. and Smith, J. A.C. (2003). Natural variation in cadmium tolerance and its relationship to metal 
hyperaccumulation for seven populations of Thlaspi caerulescens from Western Europe. Plant, Cell and Environment, (10)26: 1657-1672.

Said, S.E. (2002). Contamination of ground water with organic and inorganic pollutants in El-Sadat city, a new settlement in Egypt. M. Sc. Thesis, Fac. of Sci., Menoufia Univ., Egypt.

Salem, A.A.M. (2004). Impact of some industrial and agricultural activities of the ground water quality of Sadat city. M. Sc. Thesis, Fac. of agric., Ain Shams Univ., Egypt.

Salido, A.L.; Hasty, K.L.; Lim, J.M. and Butcher, D.J. (2003). Phytoremediation of arsenic and lead in contaminated soil using Chinese brake ferns (Pteris vittata) and Indian mustard (Brassica juncea). Inter. J. of Phytoremediation, (5)2: 89- 103.

Soltanpour, P.N. (1985). Use of ammonium bicarbonate DTPA soil tests to evaluate elemental availability and toxicity. Common, Soil Sci. Plant Anal., 16:323-318.

Still, E.R.; Mid R.J.P. and Williams. P. (1980). Potential methods for selective accumulation of nickel (II) ions by plants. Inorg. Biochem., 13:35-40.

Suresh, B. and Ravishankar, G. (2004). Phytoremediation- A Novel and promising approach for environmental clean-up. Critical Reviews in Biotechnology, (3) 24:97-124.

Turgut, C.; Pepe, M. K. and Cutright, T. (2005). The effect of EDTA on Helianthuis annuus uptake, selectivity, and translocation of heavy metals when grown in Ohio, New Mexico and Columbia Soils, Chemosphere, 58: 1087-1095.

Vazques, M.D.; Barcelo, C.H.; Poschenreider, J.; Madico, P.; Hatton, A.J. M. and Baker, G.H. (1992). Cope, localization of zinc and cadmium in Thlaspi vaerulescens (Brassicaceae), a metallophyte that can hyperaccumulate both metals. J. of Plant Physiology, 140:350-355.

Vervake, P.; Tack, F.M.G.; Navez, F.; Martin, J.; Verloo, M.G. and Lust, N. (2006). Fate of heavy metals during fixed bed downdraft gasification of willow wood harvested from contaminated sites. Biomass and Bioenergy, (30) 1: 58-65.

Yang, X.; Feng, Y.; Zhenli, H. and Stoffella, P.J. (2005). Molecular mechanisms of heavy metal hyperaccumulation and phytoremediation. J. of Trace Elements in Medicine and Biology, (18) 4: 339-353.

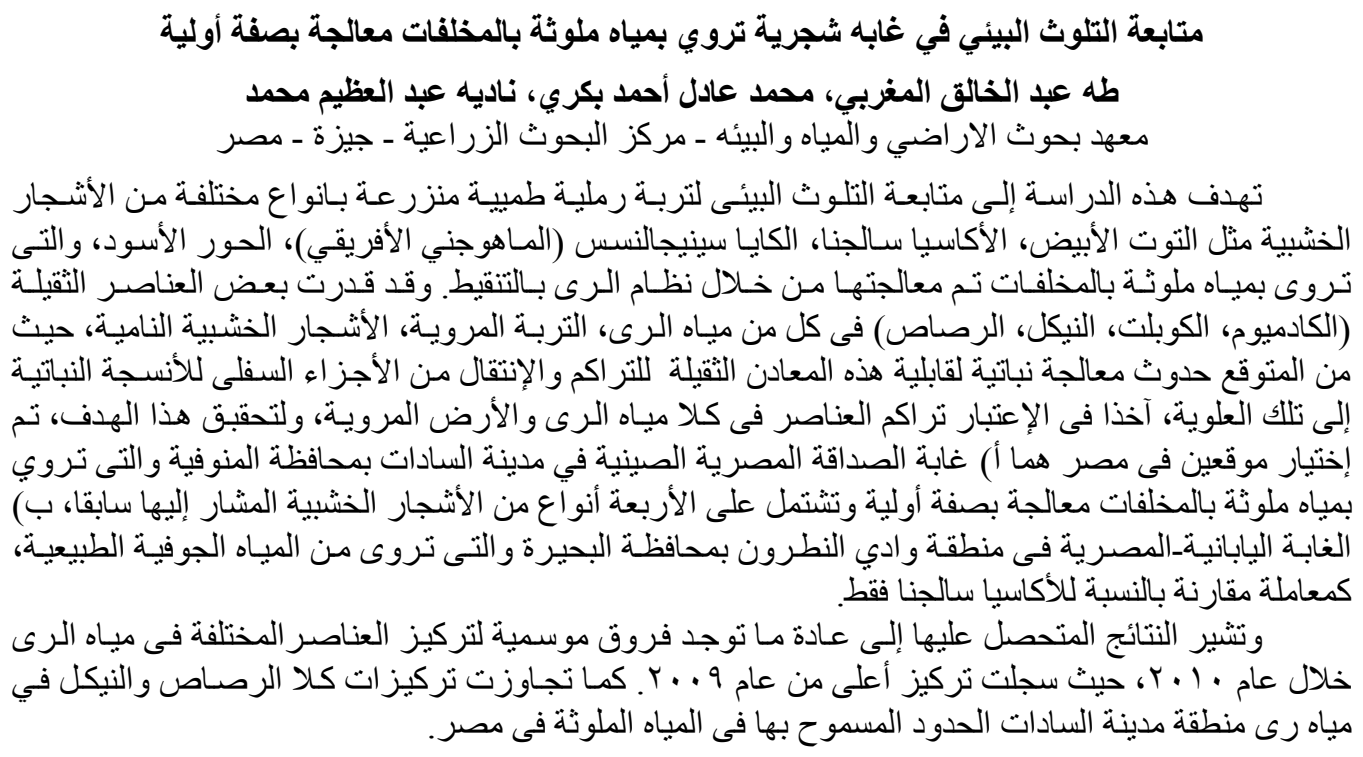

Fayoum J. Agric. Res. \& Dev., Vol.25, No.1, January, 2011 
و أيضا تجاوز تركيز النيكل فى المياه الجوفية الطبيعية بمنطقة وادى النطرون الحدود المسموح به به في مياه

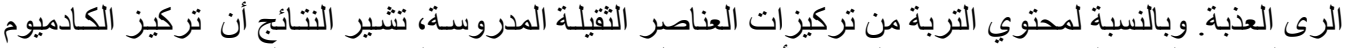

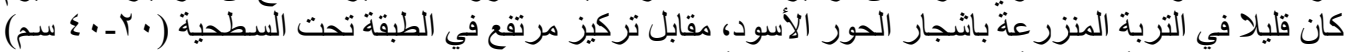

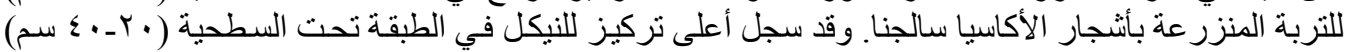

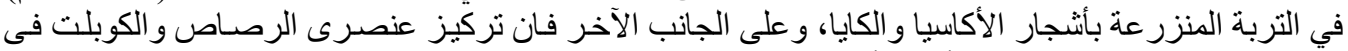

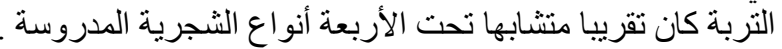

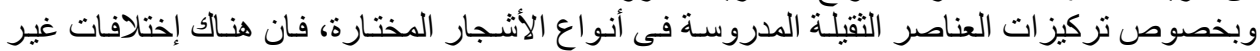

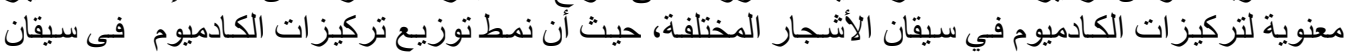

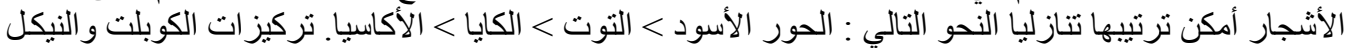

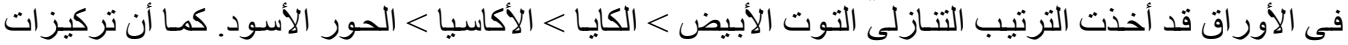

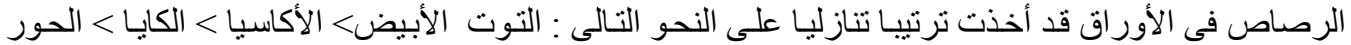

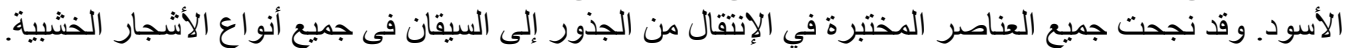

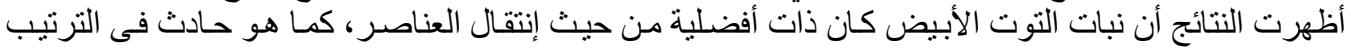

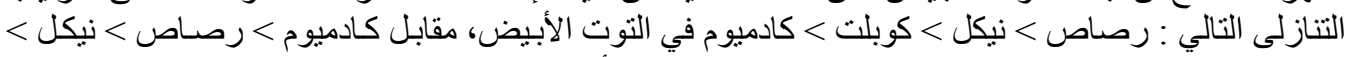

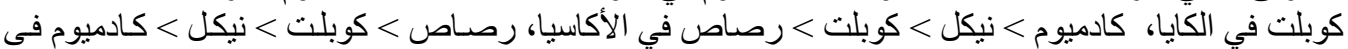

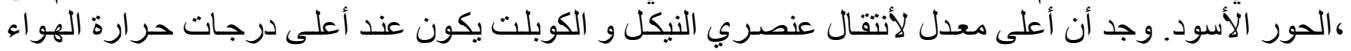

وقد وجد أن تركيز عنصرى النيكل والكوبلت، و حيث أن تركيز هذه العناصر في النبات بيتأتر بالرقم

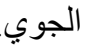

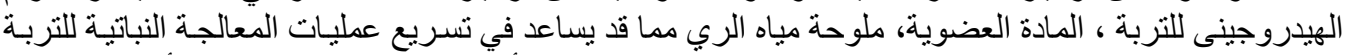

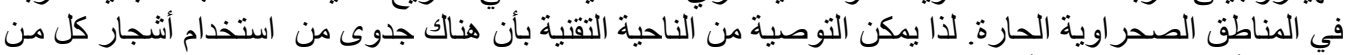

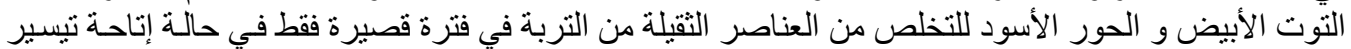

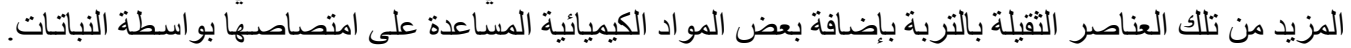

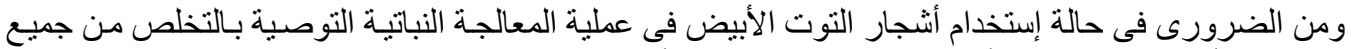

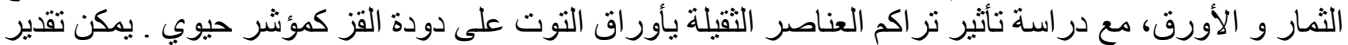

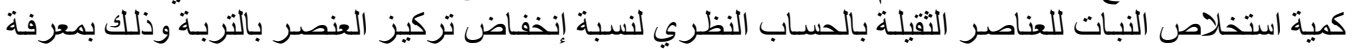

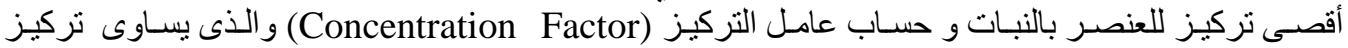

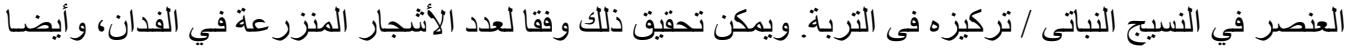

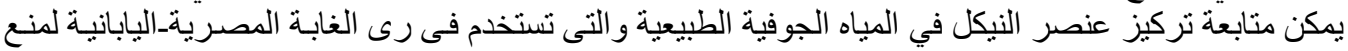

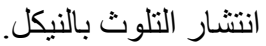

